\title{
Hematopoietic Cell Transplantation for Severe Combined Immunodeficiency Patients: a Japanese Retrospective Study
}

\author{
Satoshi Miyamoto ${ }^{1,2} \cdot$ Katsutsugu Umeda $^{2,3} \cdot$ Mio Kurata $^{4} \cdot$ Akira Nishimura $^{1,2} \cdot$ Masakatsu Yanagimachi $^{2,5}$. \\ Masataka Ishimura ${ }^{6} \cdot$ Maho Sato $^{7} \cdot$ Tomonari Shigemura $^{8} \cdot$ Motohiro Kato $^{9} \cdot$ Yoji Sasahara $^{2,10}$ - Akihiro Iguchi $^{2,11}$. \\ Takashi Koike $^{12}$ - Yoshiyuki Takahashi ${ }^{13} \cdot$ Michiko Kajiwara $^{14} \cdot$ Masami Inoue $^{7}$. Yoshiko Hashii ${ }^{15} \cdot$ Hiromasa Yabe $^{2,16}$. \\ Koji Kato $^{2,17} \cdot$ Yoshiko Atsuta ${ }^{4,18} \cdot$ Kohsuke Imai $^{2,19}$ - Tomohiro Morio ${ }^{1,2}$
}

Received: 24 November 2020 / Accepted: 25 July 2021 / Published online: 27 August 2021

(c) The Author(s), under exclusive licence to Springer Science+Business Media, LLC, part of Springer Nature 2021

\begin{abstract}
Purpose Hematopoietic cell transplantation (HCT) is a curative therapy for patients with severe combined immunodeficiency (SCID). Here, we conducted a nationwide study to assess the outcome of SCID patients after HCT in Japan.

Methods A cohort of 181 SCID patients undergoing their first allogeneic HCT in 1974-2016 was studied by using the Japanese national database (Transplant Registry Unified Management Program, TRUMP).

Results The 10-year overall survival (OS) of the patients who received HCT in 2006-2016 was 67\%. Umbilical cord blood (UCB) transplantation was performed in 81 patients $(45 \%)$. The outcomes of HCT from HLA-matched UCB $(n=21)$ and matched sibling donors $(n=22)$ were comparable, including 10 -year OS (91\% vs. $91 \%)$, neutrophil recovery (cumulative incidence at 30 days, $89 \%$ vs. $100 \%$ ), and platelet recovery (cumulative incidence at 60 days, $89 \%$ vs. $100 \%$ ). Multivariate analysis of the patients who received HCT in 2006-2016 demonstrated that the following factors were associated with poor OS: bacterial or fungal infection at HCT (hazard ratio (HR): $3.8, P=0.006$ ), cytomegalovirus infection prior to HCT (HR: 9.4, $P=0.03$ ), $\geq 4$ months of age at HCT (HR: 25.5, $P=0.009$ ), and mismatched UCB (HR: 19.8, $P=0.01$ ).

Conclusion We showed the potential of HLA-matched UCB as a donor source with higher priority for SCID patients. We also demonstrated that early age at HCT without active infection is critical for a better prognosis, highlighting the importance of newborn screening for SCID.
\end{abstract}

Keywords Severe combined immunodeficiency · Hematopoietic cell transplantation - Cord blood transplantation . Newborn screening $\cdot$ Retrospective study $\cdot$ Japan

$\begin{array}{ll}\text { Abbreviations } \\ \text { ADA } & \text { Adenosine deaminase } \\ \text { CI } & \text { Confidence interval } \\ \text { CMV } & \text { Cytomegalovirus } \\ \text { GVHD } & \text { Graft-versus-host disease } \\ \text { HR } & \text { Hazard ratio } \\ \text { HCT } & \text { Hematopoietic cell transplantation } \\ \text { IS } & \text { Immunosuppression } \\ \text { IUIS } & \text { International Union of Immunological Societies } \\ \text { MCB } & \text { Matched umbilical cord blood } \\ \text { mMCB } & \text { Mismatched umbilical cord blood } \\ \text { MSD } & \text { Matched sibling donor } \\ \text { NBS } & \text { Newborn screening }\end{array}$

$\begin{array}{ll}\text { OS } & \text { Overall survival } \\ \text { ORD } & \text { Other related donor } \\ \text { PID } & \text { Primary immunodeficiency } \\ \text { SCID } & \text { Severe combined immunodeficiency } \\ \text { TBI } & \text { Total body irradiation } \\ \text { TRUMP } & \text { Transplant Registry Unified Management } \\ & \text { Program } \\ \text { UCB } & \text { Umbilical cord blood } \\ \text { UCBT } & \text { Umbilical cord blood transplantation } \\ \text { UBM } & \text { Unrelated bone marrow } \\ \text { VOD } & \text { Veno-occlusive disease }\end{array}$

Kohsuke Imai

kimai.ped@tmd.ac.jp

Extended author information available on the last page of the article 


\section{Introduction}

Severe combined immunodeficiency (SCID) is a hereditary disorder characterized by the severe dysfunction of cellular and humoral immunity owing to impaired $\mathrm{T}$ cell and $\mathrm{B}$ cell development or function. SCID comprises heterogeneous diseases caused by abnormalities in genes related to cytokine signaling, $\mathrm{T}$ cell receptor, VDJ recombination, and metabolism [1]. To date, 17 different diseases have been classified as SCID by the International Union of Immunological Societies (IUIS) [2]. Without any treatment, SCID patients develop opportunistic and/or severe infections during infancy, which normally leads to death within the first year.

Hematopoietic cell transplantation (HCT) has been widely applied as a curative therapy for patients with SCID since 1968 [3]. Particularly, in Japan, the first registered HCT for SCID was conducted in 1974. Upon the establishment of the Japanese Cord Blood Bank Network in 1999, umbilical cord blood (UCB) is commonly used in HCT for primary immunodeficiencies (PIDs) in Japan. Especially, umbilical cord blood transplantation (UCBT) can be prepared quickly and is considered beneficial for SCID patients, many of whom require relatively urgent transplantation. We previously analyzed 40 patients with SCID undergoing UCBT, showing its feasibility for SCID patients (5-year overall survival (OS); 71\%) [4]. Although several other studies were reported from Japan [5-7], no Japanese study directly compared the outcomes of HCT for SCID patients between UCB and other donor sources. From Europe, Fernandes et al. demonstrated the transplant outcomes of SCID patients after HCT from UCB and mismatched related donors, showing similar 5-year OS $(57 \% \pm 6 \%$ vs. $62 \% \pm 4 \%$, respectively) and higher incidence of graft-versus-host disease (GVHD) in UCBT [8]. Since then, the utility of UCB as an alternative donor source has not been updated. In the present study, we conducted a Japanese nationwide retrospective analysis of SCID patients following HCT for the first time and described the characteristics of these patients, outcomes, and factors contributing to the survival of SCID patients, where nationwide newborn screening (NBS) is not available. We also demonstrated equivalent outcomes of HCT from HLA-matched UCB to those from matched sibling donors (MSDs), highlighting the potential of matched $\mathrm{UCB}$ as a donor source with higher priority for SCID patients.

\section{Methods}

\section{Data Collection}

This study was approved by the Institutional Ethics Committee of the Japan Society for Hematopoietic Cell Transplantation and Tokyo Medical and Dental University (approval number: M2018-067). The participants (and/ or their guardians) provided written informed consent for research use of their data and were registered in the Transplant Registry Unified Management Program (TRUMP), the electronic database of all HCTs performed in Japan established by the Japanese Society of Hematopoietic Cell Transplantation [9]. Patients with SCID who underwent their first HCT were included. The diagnoses of the patients were collected according to the IUIS 2017 classification [10]. All transplant data were obtained from the TRUMP. Of note, the TRUMP database does not contain the data on the following: NK cell status at diagnosis, PK monitoring of alkylating agents, lineage-specific chimerism, and immunoglobulin supplementation after HCT.

\section{Study Endpoints}

The conditioning regimens were classified as follows: fludarabine and busulfan (herein, Flu/Bu), fludarabine and melphalan (Flu/Mel), no chemotherapy (no conditioning), immunosuppression (IS, regimens containing one or more of the following: fludarabine, cyclophosphamide, anti-thymocyte globulin), and others (e.g., busulfan and cyclophosphamide [Bu/CY], cyclophosphamide and low-dose total body irradiation [TBI]). The conditioning regimens other than no conditioning or IS were also classified into myeloablative conditioning (MAC) and reducedintensity conditioning (RIC). Regimens containing one of the following were classified as MAC: TBI at a total dose of $\geq 700 \mathrm{cGy}$, busulfan at a total dose of $\geq 12 \mathrm{mg} / \mathrm{kg}$, or melphalan at a total dose of $>140 \mathrm{mg} / \mathrm{m}^{2}$, according to previous studies from North America [11, 12].

The definition of donor types is as follows: MSD, other related donor (ORD), matched cord blood (MCB), mismatched cord blood (mMCB), and unrelated bone marrow (UBM). HLA matching was determined by serology for earlier patients and was defined by genotype in more recent periods (among non-MSD recipients, 25\% were determined by genotype before 2006 vs. $91 \%$ in 2006-2016). HLA-A, HLA-B, and HLA-DRB1 were registered in the 
database for most of the cases, whereas HLA-C was only searched in the more recent patients. To strictly classify the HLA disparity, we used the term "matched" to refer to those $8 / 8$ matched or $6 / 6$ matched patients who lacked the HLA-C locus data (Table S1). Neutrophil recovery is defined as the achievement of an absolute neutrophil count of $\geq 0.5 \times 10^{9} / \mathrm{L}$ for 3 consecutive days. Platelet recovery was defined as the achievement of an absolute platelet count of $\geq 50 \times 10^{9} / \mathrm{L}$ for 3 consecutive days, unsupported by transfusion for 7 days. Retransplantation refers to both "boost" (reinfusion of stem cells from the same donor without conditioning) and "second HCT" (reinfusion of stem cells from a different donor with or without conditioning, or reinfusion from the same donor with conditioning). Whole blood cell chimerism was evaluated from 100 days to 1.5 years after HCT and the patients who died before achieving neutrophil recovery were excluded. We classified chimerism as follows: complete donor $(\geq 95 \%$ donor chimerism), donor dominant ( $<95 \%$ and $\geq 80 \%$ donor chimerism), mixed chimerism ( $<80 \%$ and $\geq 20 \%$ donor chimerism), and low chimerism $(<20 \%$ donor chimerism or patients who required retransplantation). We defined short stature as a height at least 2 standard deviations below the mean height and gonadal dysfunction as an abnormality in either laboratory tests for gonadal hormones or semen analyses, or irregularity or absence of menstruation.

\section{Statistical Analysis}

Retransplantation, neutrophil recovery, platelet recovery, acute GVHD (aGVHD), and chronic GVHD (cGVHD) were analyzed using a cumulative incidence method. Death before retransplantation was considered a competing event for retransplantation. Death or retransplantation before neutrophil recovery was considered a competing event for neutrophil recovery. For platelet recovery, aGVHD, or cGVHD, death or retransplantation was considered a competing event as well. In the analyses for neutrophil and platelet recovery, we excluded patients who received no conditioning or IS. We calculated the cumulative incidence of cGVHD, limiting to the patients who survived more than 100 days after HCT. Gray's test was used for the group comparisons of cumulative incidence. OS was calculated using Kaplan-Meier estimates, and Cox proportional hazard models were used to evaluate the impact of the independent risk factors on OS. Donor type, disease phenotype, age at HCT, bacterial and fungal infection at HCT, cytomegalovirus (CMV) infection prior to HCT, and conditioning regimen were considered variables because these factors have significant effects on OS according to previous reports [11-13]. For the multivariate analysis, we limited the patients to those who received HCT from 2006 onwards, this was when there were few missing values for each variable. The cumulative incidence, OS, and hazard ratios (HRs) are reported with 95\% confidence intervals (CIs). All statistical analyses were performed using Stata software v16.1 and EZR 1.42 [14] Two-sided $p<0.05$ was considered significant. All values enclosed in brackets [] represent $95 \%$ CIs.

\section{Results}

\section{Patient Characteristics}

A total of 181 SCID patients comprising 144 males (80\%) and 37 females (20\%) who underwent HCT between 1974 and 2016 were included in this study (a median time between HCT and last follow-up: 3.7 years; 1 day-28.5 years). The patient characteristics are shown in Table 1 . The subtypes of SCID were identified in 100 participants (55\%), with $I L 2 R G$ as the most common, accounting for 55 patients (55\%). Autosomal recessive SCID was relatively rare, reflecting the low rate of consanguineous marriage in Japan. The patients were diagnosed at a median age of 5 months (0 months-16 years) without improvement over time (Table S2) and received HCT at a median age of 7 months ( 1 month-17 years), with 65 days ( 0 days-11.0 years) as a median time between diagnosis and HCT.

HCT from related donors accounted for 89 cases (49\%), whereas those from MSDs included 22 cases (12\%). The remaining 92 cases $(51 \%)$ were from unrelated donors, 81 (45\%) of which were from UCB. Among the 81 cases receiving UCBT, 21 (12\%) cases were from MCB. All UCBTs were single-unit unrelated cord blood transplantations. Once patients were diagnosed, UCBT could be performed as soon as HCT from related donor, whereas it took longer to perform HCT from URBM (Table S3). UCB became the most common donor source for SCID transplants in Japan, accounting for 53 cases (70\%) in 2006-2016 (Table S2). The conditioning regimens and GVHD prophylaxes changed over time. More patients received Flu/Bu or Flu/Mel for conditioning and tacrolimus for GVHD prophylaxis in the later period (Table S2).

Notably, 33 patients (33\%) had an active bacterial or fungal infection at the time of HCT. Among those who received HCT in 2006-2016, active bacterial or fungal infection at HCT was correlated with the following conditions (Table S4): a shorter period between diagnosis and HCT ( 80 days for those without infection vs. 33 days for those with infection; $P=0.001$ ), history of mechanical ventilation before HCT (11\% for those without infection vs. $33 \%$ for those with infection; $P=0.03$ ), and respiratory impairment at HCT (26\% for those without infection vs. $56 \%$ for those with infection; $P=0.02$ ). The patients who received no conditioning tended to be commonly chosen in the patients 
Table 1 Details of the patient characteristics

Characteristics (number of patients evaluated)

Patient sex $(n=181)$

Male $144(80 \%)$

Female $37(20 \%)$

Phenotype $(n=100)$

$\mathbf{T}-\mathbf{B}+\mathbf{S C I D}$

$I L 2 R G$ deficiency/X-linked

$J A K 3$ deficiency

$65(65 \%)$

$55(55 \%)$

CD3 component deficiency

Unspecified $\mathrm{T}-\mathrm{B}+\mathrm{SCID}$

T-B - SCID

RAG1/RAG2 deficiency/Omenn syndrome

ADA deficiency

Artemis deficiency

DNA ligase IV deficiency

Reticular dysgenesis

Unspecified T - B - SCID

Age at diagnosis $(n=173)$

$<3$ months

$\geqq 3$ months

Median (range)

Age at HCT $(n=175)$

$<4$ months

$\geqq 4$ months

Median (range)

Time from diagnosis to HCT $(n=175)$

$\sim 3$ months

$3 \sim 6$ months

$6 \sim$ months

Median interval

Year of HCT $(n=181)$

$1974 \sim 2005$

2006 2016

Bacterial or fungal infection at HCT $(n=99)$

Yes

No

GVHD prophylaxis $(n=158)$

Cyclosporine

Tacrolimus

Conditioning $(n=169)$

Flu/Bu

Flu/Mel ${ }^{\mathrm{a}}$

No conditioning

Immunosuppression

$\mathrm{Flu} / \mathrm{CY}+\mathrm{ATG}$

Flu

CY

ATG

Others

$\mathrm{Bu} / \mathrm{CY}$

Flu/CY/ETP/Mel

$33(33 \%)$

$66(64 \%)$

$87(55 \%)$

$71(45 \%)$

$28(17 \%)$

$34(20 \%)$

$72(43 \%)$

$7(4 \%)$

$3(2 \%)$

$2(1 \%)$

$1(0.6 \%)$

$28(17 \%)$

$18(11 \%)$
Table 1 (continued)

Characteristics (number of patients evaluated)

\begin{tabular}{ll} 
Flu/Bu/Mel & $2(1 \%)$ \\
Flu + low-dose TBI & $2(1 \%)$ \\
Flu/CY + low-dose TBI & $1(0.6 \%)$ \\
TBI 8 Gy/CY & $1(0.6 \%)$ \\
AraC/ETP/CY & $1(0.6 \%)$ \\
n vivo T cell depletion $(\boldsymbol{n = 1 6 6 )}$ & \\
Yes & $23(14 \%)$ \\
No & $143(86 \%)$ \\
Donor type (n=181) & \\
BM & $\mathbf{9 1 ( 5 0 \% )}$ \\
MSD & $22(12 \%)$ \\
MORD & $5(3 \%)$ \\
mMORD & $53(29 \%)$ \\
UBM & $11(6 \%)$ \\
UCB & $\mathbf{8 1}(\mathbf{4 5} \%)$ \\
MCB (unrelated) & $21(12 \%)$ \\
mMCB (unrelated) & $59(33 \%)$ \\
NA (unrelated) & $1(0.6 \%)$ \\
PB & $\mathbf{9 ( 5 \% )}$ \\
MORD & $1(0.6 \%)$ \\
mMORD & $7(4 \%)$ \\
NA (related) & $1(0.6 \%)$ \\
\hline
\end{tabular}

${ }^{\mathrm{a}}$ Among Flu/Mel, 4 patients received Flu/Mel combined with lowdose TBI (3 Gy)

$S C I D$, severe combined immunodeficiency; $A D A$, adenosine deaminase; $H C T$, hematopoietic cell transplantation; $G V H D$, graft-versushost disease; Flu, fludarabine; $\mathrm{Bu}$, busulfan; $\mathrm{Mel}$, melphalan; TBI, total body irradiation; $C Y$, cyclophosphamide; $A T G$, anti-thymocyte globulin; ETP, etoposide; AraC, cytarabine; $B M$, bone marrow; $M S D$, matched sibling donor; $M O R D$, matched other related donor; mMORD, mismatched other related donor; $U B M$, unrelated bone marrow; $U C B$, umbilical cord blood; $M C B$, matched cord blood; $m M C B$, mismatched cord blood; $N A$, not applicable; $P B$, peripheral blood

$1(0.6 \%)$

$3(2 \%)$ with an active infection at HCT, although it did not reach significance $(P=0.06)$.

\section{Survival and Causes of Death}

The summary of the transplant outcomes is shown in Table 2. The OS for 25 years was 44\% [23-63\%], and the median time of death from HCT was 51 days (1 day-24.3 years). The 10 -year OS was 67\% [55-77\%] in 2006-2016 vs. 55\% [45-64\%] in 1974-2005 (Fig. 1a; $P=0.14$ ); the number of patients per period was low, and the difference did not reach significance.

Based on univariate analyses, there were significant differences in OS between the following: age at HCT (10 years: $84 \%$ [63-94\%] for $<4$ months vs. 56\% [48-64\%] for $\geq 4$ months, Fig. 1b, $P=0.02$ ); bacterial and fungal 
Table 2 Comparisons of the outcomes by the period of HCT

\begin{tabular}{|c|c|c|c|c|}
\hline Outcome (number of patients evaluated) & Overall & $\sim 2005$ & $2006 \sim$ & $P$ value \\
\hline Overall survival $(\%)(n=181)$ & & & & $0.14^{\mathrm{a}}$ \\
\hline 1 year $(95 \% \mathrm{CI})$ & $70(62-76)$ & $65(55-73)$ & $76(65-84)$ & \\
\hline 5 years $(95 \% \mathrm{CI})$ & $61(53-67)$ & $56(46-65)$ & $67(55-77)$ & \\
\hline 10 years $(95 \% \mathrm{CI})$ & $59(51-66)$ & $55(45-64)$ & $67(55-77)$ & \\
\hline 25 years $(95 \% \mathrm{CI})$ & $44(23-63)$ & $41(21-59)$ & NA & \\
\hline Retransplantation $(n=181)$ & & & & $0.47^{\mathrm{b}}$ \\
\hline Cumulative incidence (\%) at 10 years $(95 \% \mathrm{CI})$ & $9(6-14)$ & $11(6-17)$ & $9(3-19)$ & \\
\hline Median days of retransplantation (range) & $126.5(31-2225)$ & $125(33-1323)$ & $151(31-2225)$ & \\
\hline Neutrophil recovery $(n=90)^{\mathrm{c}}$ & & & & $0.84^{\mathrm{b}}$ \\
\hline Cumulative incidence $(\%)$ at 30 days $(95 \% \mathrm{CI})$ & $78(68-85)$ & $78(60-89)$ & $78(64-87)$ & \\
\hline Cumulative incidence (\%) at 42 days $(95 \% \mathrm{CI})$ & $83(74-90)$ & $81(62-91)$ & $85(72-93)$ & \\
\hline Median days of recovery (range) & $17(0-49)$ & $16(11-49)$ & $17(0-37)$ & \\
\hline Platelet recovery $(n=82)^{\mathrm{c}}$ & & & & $0.17^{\mathrm{b}}$ \\
\hline Cumulative incidence (\%) at 30 days (95\% CI) & $49(38-59)$ & $35(18-52)$ & $57(42-69)$ & \\
\hline Cumulative incidence $(\%)$ at 60 days $(95 \% \mathrm{CI})$ & $73(62-82)$ & $66(45-80)$ & $77(63-87)$ & \\
\hline Median days of recovery (range) & $28(0-153)$ & $36.5(6-153)$ & $26(0-129)$ & \\
\hline \multicolumn{5}{|l|}{ Acute GVHD } \\
\hline \multicolumn{5}{|l|}{ Cumulative incidence $(\%)$ at 1 year $(95 \% \mathrm{CI})$} \\
\hline Grades II-IV $(n=167)$ & $16(11-22)$ & $13(7-21)$ & $20(12-30)$ & $0.28^{\mathrm{b}}$ \\
\hline Grades III-IV $(n=173)$ & $6(3-11)$ & $5(2-11)$ & $8(3-15)$ & $0.46^{\mathrm{b}}$ \\
\hline \multicolumn{5}{|l|}{ Chronic GVHD } \\
\hline \multicolumn{5}{|l|}{ Cumulative incidence $(\%)$ at 2 years $(95 \% \mathrm{CI})$} \\
\hline Total $(n=125)$ & $12(7-19)$ & $10(4-19)$ & $15(7-25)$ & $0.5^{\mathrm{b}}$ \\
\hline Extensive $(n=127)$ & $6(3-12)$ & $4(1-11)$ & $9(3-18)$ & $0.28^{\mathrm{b}}$ \\
\hline
\end{tabular}

${ }^{\mathrm{a}}$ Logrank test

${ }^{\mathrm{b}}$ Gray's test

${ }^{\mathrm{c}}$ The patients who received unconditioned HCT or immunosuppression were excluded from the analysis for hematologic recovery

$H C T$, hematopoietic cell transplantation; $C I$, confidence interval; $N A$, not applicable; $G V H D$, graft-versus-host disease

infection at HCT (10 years: 75\% [62-84\%] for those without infection vs. 50\% [31-66\%] for those with infection, Fig. 1c, $P=0.008$ ); CMV infection prior to HCT (5 years: $71 \%$ [60-79\%] for those without infection vs. 33\% [8-62\%] for those with infection, Fig. 1d, $P<0.001)$; and conditioning regimen (10 years: $80 \%$ [68-88\%] for RIC, 55\% [31-74\%] for MAC, and 51\% [39-61\%] for no conditioning/IS, Fig. 1e, $P=0.007)$. There was no difference in OS between the Flu/ $\mathrm{Bu}$ and $\mathrm{Flu} / \mathrm{Mel}$ regimens, with better survival than those who received no conditioning/IS or other regimens (Fig. 1f, $P=0.002)$. Meanwhile, the SCID phenotype or genotype did not show a significant difference in OS (Fig. 1g, h). The 10-year OS of the HCT cases from MSD (91\% [68-98\%]) and those from MCB (91\% [67-98\%]) were similar, having better survival than the other donor type (Fig. 1i).

We additionally performed univariate analyses on the OS of patients who received HCT from 2006 onwards. Although these analyses may be less accurate owing to the smaller sample size, similar trends were observed for each variable (Table 3).

Multivariate analysis of the patients who received HCT from 2006 onwards revealed that age at HCT $\geq 4$ months (HR: 25.5 [2.2-293]; $P=0.009$ ), mMCB (HR: 19.8 [2.0-197]; $P=0.01)$, bacterial and fungal infection at HCT (HR: 3.8 [1.5-9.8]; $P=0.006$ ), CMV infection prior to HCT (HR: 9.4 [1.3-66.4]; $P=0.03$ ), and no conditioning/IS (HR: 11.7 [2.7-50.7]; $P=0.001$ ) were associated with worse OS (Table 3). The SCID phenotype did not significantly affect OS.

The causes of death are shown in Table 4. Infection and non-infectious pulmonary complications were frequent, accounting for 27 cases (37\%) and 15 cases (21\%), respectively. The number of deaths due to infection was higher in the group with no conditioning ( $P=0.05$, Table S5). 


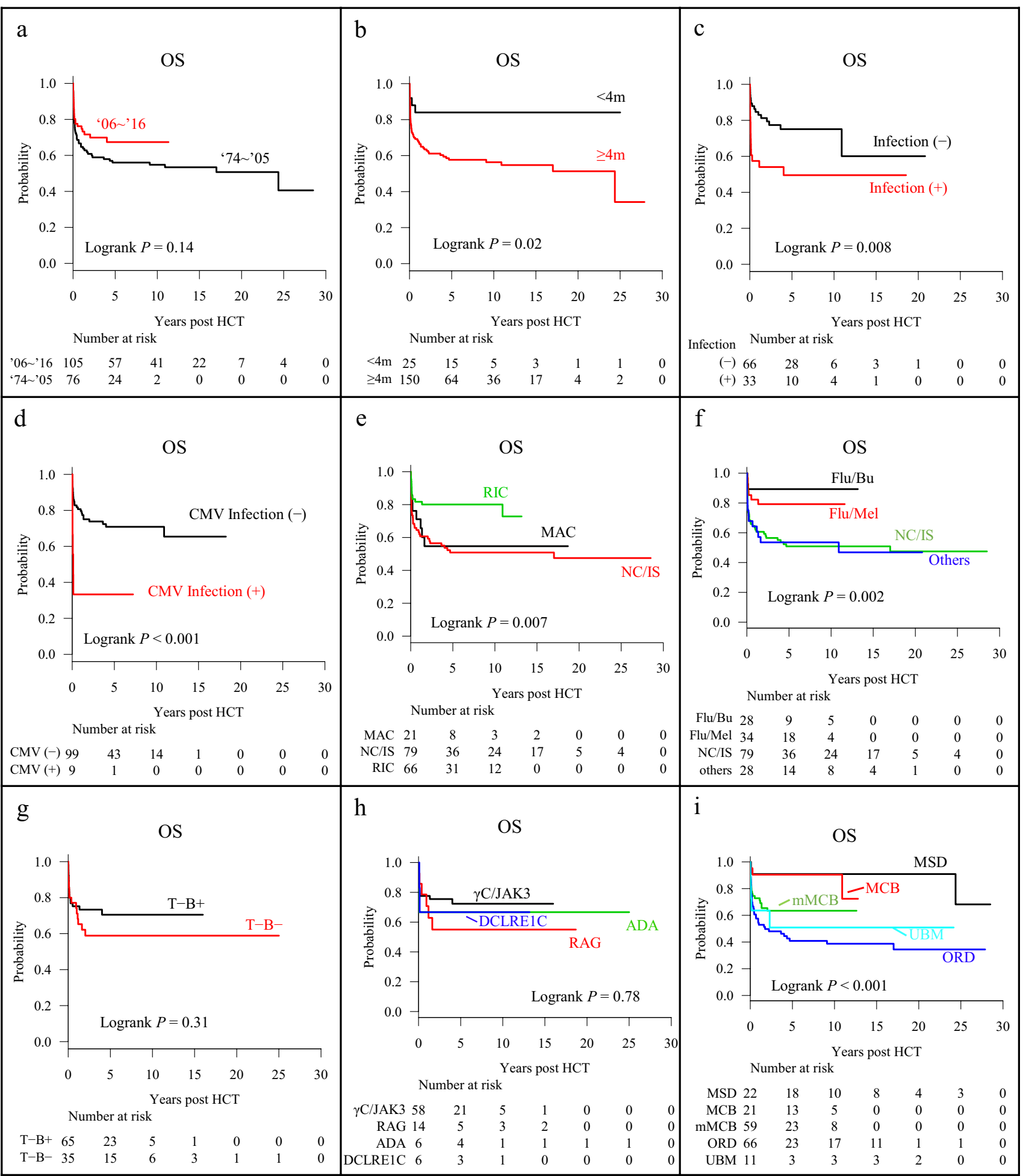

Fig. 1 Kaplan-Meier survival curves. a OS according to the period when transplanted. The subsequent analyses for OS were applied to the patients in all periods, according to $\mathbf{b}$ age at HCT diagnosis, $\mathbf{c}$ the presence of bacterial or fungal infection status at HCT, d CMV infection prior to HCT, e intensity of conditioning, $\mathbf{f}$ conditioning regimen, $\mathbf{g}$, h SCID phenotype, and $\mathbf{i}$ donor type. OS, overall survival;
$H C T$, hematopoietic cell transplantation; $C M V$, cytomegalovirus; $R I C$, reduced-intensity conditioning; $M A C$, myeloablative conditioning, $N C$, no conditioning; $I S$, immunosuppression; Flu, fludarabine; $B u$, busulfan; $M e l$, melphalan; $M S D$, matched sibling donor; $M C B$, matched cord blood; $m M C B$, mismatched cord blood; ORD, other related donor; $U B M$, unrelated bone marrow 
Table 3 Factors affecting OS after HCT between 2006 and 2016

\begin{tabular}{|c|c|c|c|c|c|}
\hline \multirow[t]{2}{*}{ Factors } & \multirow[t]{2}{*}{$n$} & \multicolumn{2}{|l|}{ Univariate analysis } & \multicolumn{2}{|l|}{ Multivariate analysis } \\
\hline & & 5-year OS (95\% CI) & $P$ value & Hazard ratio $(95 \% \mathrm{CI})$ & $P$ value \\
\hline SCID phenotype & & & 0.45 & & \\
\hline $\mathrm{T}-\mathrm{B}+\mathrm{SCID}$ & 53 & $69 \%(53-81 \%)$ & & 1 & \\
\hline $\mathrm{T}-\mathrm{B}-\mathrm{SCID}$ & 21 & $60 \%(35-78 \%)$ & & $0.37(0.12-1.1)$ & 0.08 \\
\hline Age at HCT & & & 0.046 & & \\
\hline$<4 \mathrm{~m}$ & 14 & $93 \%(59-99 \%)$ & & 1 & \\
\hline$\geq 4 \mathrm{~m}$ & 61 & $62 \%(47-74 \%)$ & & $25.5(2.2-293)$ & 0.009 \\
\hline Donor type & & & 0.61 & & \\
\hline MSD & 7 & $86 \%(33-98 \%)$ & & 1 & \\
\hline MCB (unrelated) & 13 & $85 \%(51-96 \%)$ & & $7.4(0.5-100)$ & 0.13 \\
\hline mMCB (unrelated) & 39 & $66 \%(48-78 \%)$ & & $19.8(2.0-197)$ & 0.01 \\
\hline ORD & 11 & $48 \%(9-80 \%)$ & & $5.9(0.6-54.7)$ & 0.12 \\
\hline UBM & 5 & NA & & $22.3(1.4-357)$ & 0.03 \\
\hline Bacterial or fungal infection at HCT & & & 0.005 & & \\
\hline No & 47 & $79 \%(64-89 \%)$ & & 1 & \\
\hline Yes & 27 & $49 \%(28-67 \%)$ & & $3.8(1.5-9.8)$ & 0.006 \\
\hline CMV infection prior to $\mathrm{HCT}$ & & & 0.45 & & \\
\hline No & 70 & $69 \%(56-79 \%)$ & & 1 & \\
\hline Yes & 5 & $60 \%(13-88 \%)$ & & $9.4(1.3-66.4)$ & 0.03 \\
\hline Conditioning & & & 0.04 & & \\
\hline $\mathrm{Flu} / \mathrm{Bu}$ & 22 & $86 \%(63-95 \%)$ & & 1 & \\
\hline Flu/Mel & 26 & $77 \%(55-89 \%)$ & & $2.0(0.5-8.5)$ & 0.33 \\
\hline No conditioning/immunosuppression & 21 & $41 \%(18-63 \%)$ & & $11.7(2.7-50.7)$ & 0.001 \\
\hline Others & 6 & $67 \%(20-90 \%)$ & & $3.6(0.5-24.1)$ & 0.2 \\
\hline
\end{tabular}

$O S$, overall survival; $C I$, confidence interval; $S C I D$, severe combined immunodeficiency; $H C T$, hematopoietic cell transplantation; $M S D$, matched sibling donor; $M C B$, matched cord blood; $m M C B$, mismatched cord blood; $O R D$, other related donor; $U B M$, unrelated bone marrow; $N A$, not applicable; $C M V$, cytomegalovirus; Flu, fludarabine; $B u$, busulfan; $M e l$, melphalan

\section{Hematologic Recovery and Retransplantation}

The cumulative incidence of neutrophil and platelet recovery did not differ over time (Table 2). The HCT from MSD showed the fastest hematologic recovery (Fig. 2a, b). MCB also showed relatively robust neutrophil recovery (cumulative incidence at 30 days; $89 \%$ [57-98\%]) and platelet recovery (cumulative incidence at 60 days; $89 \%$ [57-98\%]). Retransplantation was performed in 16 cases $(9 \%)$ with an interval from the HCT of 126.5 days (31 days-6.1 years). We showed an increased incidence of retransplantation in the HCT from ORD and MMCB (Fig S2a). However, no case required retransplantation after the HCT from MCB, suggesting substantially stable engraftment of MCB. Among the UCBT cases, HLA disparity tended to result in slower platelet recovery and increased incidence of retransplantation (Fig. 2b, Fig. S1c, and Fig. S2a, b).

In terms of conditioning regimens, the Flu/Bu and Flu/ Mel regimens similarly showed robust hematologic recovery (Fig. 2c, d), as well as a low incidence of retransplantation in these groups (Fig. S2c).

\section{Chimerism}

The whole blood cell chimerism according to donor type is shown in Fig S3a. Among patients with HCT from MCB, $67 \%$ had donor chimerism of $80 \%$ or higher, while none had donor chimerism of less than $20 \%$. Chimerism according to a type of conditioning regimen is also shown in Fig. S3b. Complete or donor-dominant chimerism was significantly more frequently seen in patients who received RIC than no conditioning/IS $(P=0.01)$. Among RIC regimens, complete or donor-dominant chimerism might have been more frequent in $\mathrm{Flu} / \mathrm{Bu}$ than Flu/Mel, although the difference was not significant.

\section{HCT-Related Complications}

The cumulative incidences of aGVHD II-IV and cGVHD did not change over time (Table 2). In the HCT from MSD, there was no occurrence of grades II-IV aGVHD or cGVHD. The HCT from MCB or mMCB showed similar incidence of GVHD to that from ORD (grades II-IV and grades III-IV 
Table 4 Causes of death

\begin{tabular}{ll}
\hline Cause of death & $n(\%)$ \\
\hline Infection & $27(37 \%)$ \\
Non-infection & $46(63 \%)$ \\
Pulmonary (non-infection) & $15(21 \%)$ \\
Others (non-infection) & $31(42 \%)$ \\
Veno-occulusive disease & $4(5 \%)$ \\
Unknown & $4(5 \%)$ \\
Multi-organ failure & $3(4 \%)$ \\
Cardiac failure/cardiomyopathy & $3(4 \%)$ \\
Secondary malignancy & $2(3 \%)$ \\
Renal failure & $2(3 \%)$ \\
CNS dysfunction & $2(3 \%)$ \\
Acute GVHD & $2(3 \%)$ \\
Chronic GVHD & $2(3 \%)$ \\
Adrenal & $2(3 \%)$ \\
Hemorrhage & $2(3 \%)$ \\
Liver failure & $1(1 \%)$ \\
Hemophagocytic syndrome & $1(1 \%)$ \\
Thrombotic microangiopathy & $1(1 \%)$ \\
total & 73 \\
\hline
\end{tabular}

$C N S$, central nervous system; $G V H D$, graft-versus-host disease

aGVHD at 1 year: 19\% [6-38\%] and 10\% [2-27\%] for MCB vs. $21 \%[11-32 \%]$ and $12 \%$ [5-22\%] for mMCB vs. $16 \%$ [8-27\%] and 3\% [0.6-10\%] for ORD, respectively; total and extensive cGVHD at 2 years: $6 \%$ [0.3-23\%] and $6 \%$ [0.3-23\%] for MCB vs. $22 \%$ [11-36\%] and $12 \%$ [4-24\%] for $\mathrm{mMCB}$ vs. $13 \%$ [5-25\%] and 5\% [0.9-15\%] for ORD, respectively; Fig. 2e-h). Among the UCBT cases, HLA disparity showed a trend towards more frequent GVHD occurrence (Fig. S1d-g), although it was not statistically significant. A similar trend was observed between GVHD and donor type in HCT cases performed after 2006 (data not shown).

The other HCT-related complications identified are listed in Table 5. CMV infection was observed in $15 \%$ of the patients. Among the various infections that complicated during the pre- or post-transplant course, CMV infection was correlated with poorer OS $(P<0.001$; Table S6). However, other viral infections were not associated with poor OS. The number of evaluable patients was small, and we could not show an association of donor type selection and viral clearance or occurrence of viral infection after HCT (data not shown).

Referring to the dose of busulfan, 28 patients received $\mathrm{Flu} / \mathrm{Bu}$ regimen with a median busulfan dose of $9.9 \mathrm{mg} /$ $\mathrm{kg}$ (4-19 mg/kg), among which only four patients received more than $12 \mathrm{mg} / \mathrm{kg}$ of busulfan, which was considered to be myeloablative. In contrast, 18 patients received $\mathrm{Bu} / \mathrm{CY}$ regimen with a median busulfan dose of $16 \mathrm{mg} / \mathrm{kg}(8-20 \mathrm{mg} /$ $\mathrm{kg}$ ). Veno-occlusive disease (VOD) was developed in eight patients, six of whom received $\mathrm{Bu} / \mathrm{CY}$ as the conditioning regimen (data not shown). Among 110 evaluable patients, 24 (22\%) reported short stature. The patients with short stature were significantly associated with extensive cGVHD (22\% [8-40\%] for those with short stature vs. 3\% [0.5-8\%] for those without, $P=0.002$ ). In addition, short stature was more frequently observed in patients who received "others" conditioning (Table S7; $P=0.06$ ), especially in patients receiving the $\mathrm{Bu} / \mathrm{CY}$ regimen. There was no significant association between the higher dose of alkylating agents and the higher incidence of short stature either in the Flu/Bu $(P=0.39)$ or Flu/Mel $(P=0.82)$ regimen group. However, in this registry, there were no serum concentration data of alkylating agents, which is highly variable especially in infants, and we could not analyze the association between their toxicity and the actual exposure.

\section{Discussion}

In $2006-2016,67 \%$ of HCT patients had a 10 -year OS, which is comparable to that reported elsewhere (North America [12], Europe [13], and Turkey [15]: 10-year OS, $71 \%, 71 \%$, and $66 \%$, respectively; Australia and New Zealand: 5-year OS, 70\% [16]. The HCT from MSD showed a 10 -year OS of $91 \%$, which is also consistent with these reports.

Unlike other studies, we could not detect an OS difference in OS between $\mathrm{T}-\mathrm{B}+\mathrm{SCID}$ and $\mathrm{T}-\mathrm{B}-\mathrm{SCID}$. The sample size may have been insufficient, and the influence of other factors may have been too strong to clearly assess the risk of confounding SCID phenotypes. Another reason could be differences in the distribution of $\mathrm{T}-\mathrm{B}-\mathrm{SCID}$ diseases. Among T-B - SCID, the percentage of adenosine deaminase deficiency (ADA-SCID), with a similar 10-year OS of $\sim 60 \%$ in North America and this cohort, was lower in Japan than in western countries (17.1\% in our study, whereas $35.7 \%$ in North America [11], and 25.0\% in Europe [13]). This may have reduced the negative impact of $\mathrm{T}-\mathrm{B}-\mathrm{SCID}$ on OS.

UCBT for SCID has been frequently performed in Japan, representing $70 \%$ of recent cases. This is due to accessibility to UCB, declining birth rates that lead to decreased MSD availability, and long waits for unrelated donors in Japan. In contrast, UCBT is performed less in Western countries: $11 \%$ in North America [11] and $12 \%$ of unrelated donors including UCBT in Europe [13]. Although GVHD of MCB recipients was more frequent than that of MSD, we confirmed that the survival and engraftment after HCT from $\mathrm{MCB}$ and MSD were almost equivalent.

Other studies in Western countries have shown that MCB accounted for $20-30 \%$ of unrelated UCBT [17, 18], which is 
Fig. 2 Cumulative incidence of outcomes. The cumulative incidences of neutrophil recovery and platelet recovery according to (a and $\mathbf{b}$, respectively) donor types and (c and d, respectively) conditioning regimens, and the cumulative incidences of e grades II-IV acute GVHD, f grades II-IV acute GVHD, $\mathbf{g}$ chronic GVHD, and $\mathbf{h}$ extensive chronic GVHD according to donor types are shown. HCT, hematopoietic cell transplantation; $M S D$, matched sibling donor; $M C B$, matched cord blood; $m M C B$, mismatched cord blood; $O R D$, other related donor; $U B M$, unrelated bone marrow; Flu, fludarabine; $B u$, busulfan; Mel, melphalan; $N C$, no conditioning; $I S$, immunosuppression; GVHD, graftversus-host disease

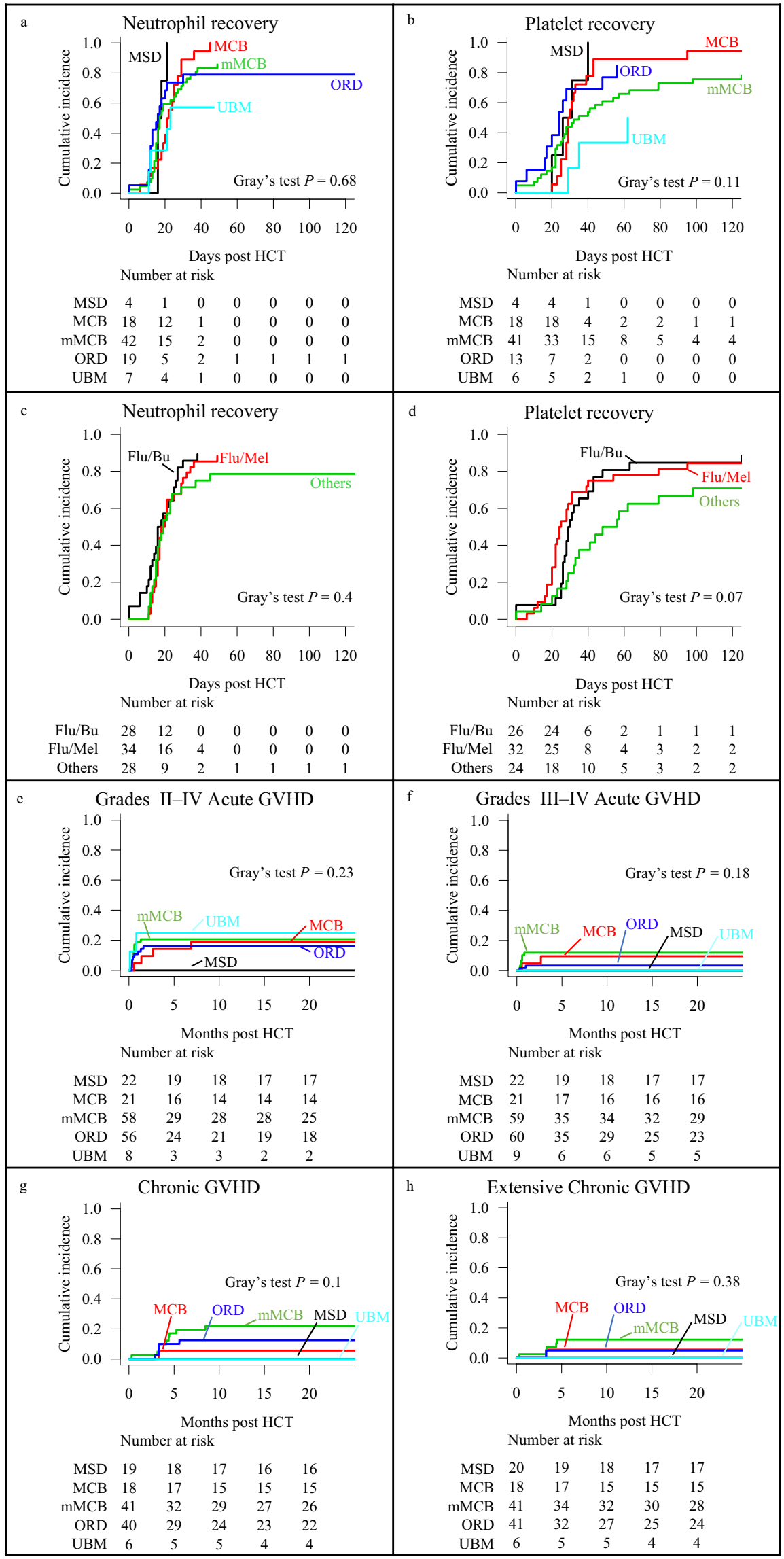


Table 5 Transplant-associated complications

\begin{tabular}{|c|c|c|}
\hline & $n(\%)$ & $\begin{array}{l}\text { Number } \\
\text { of patients } \\
\text { evaluated }\end{array}$ \\
\hline \multicolumn{3}{|l|}{ Infection } \\
\hline CMV antigenemia & $19(20 \%)$ & 96 \\
\hline CMV infection & $17(15 \%)^{\mathrm{a}}$ & 116 \\
\hline Pneumonia & $14(12 \%)^{\mathrm{a}}$ & 116 \\
\hline Hepatitis & $4(3 \%)^{\mathrm{a}}$ & 116 \\
\hline Enterocolitis & $3(3 \%)^{\mathrm{a}}$ & 116 \\
\hline Retinitis & $4(3 \%)^{\mathrm{a}}$ & 116 \\
\hline Encephalitis/meningitis & $3(3 \%)^{\mathrm{a}}$ & 116 \\
\hline Myocarditis/nephritis & $1(0.9 \%)^{\mathrm{a}, \mathrm{b}}$ & 116 \\
\hline Other viral infection & $17(15 \%)^{\mathrm{a}}$ & 111 \\
\hline Norovirus & $4(4 \%)^{\mathrm{a}}$ & 110 \\
\hline RSV & $3(3 \%)^{\mathrm{a}}$ & 110 \\
\hline EBV & $3(3 \%)^{\mathrm{a}}$ & 111 \\
\hline $\begin{array}{l}\text { HHV6/adenovirus/coronavirus/rotavi- } \\
\text { rus/VZV/Rhinovirus }\end{array}$ & $1(0.9 \%)^{\mathrm{b}}$ & 110 \\
\hline Fungal infection & $5(3 \%)^{\mathrm{c}}$ & 173 \\
\hline Candidiasis & $1(0.6 \%)^{\mathrm{c}}$ & 173 \\
\hline Aspergillosis & $4(2 \%)^{\mathrm{c}}$ & 173 \\
\hline Bacterial infection & $28(26 \%)^{\mathrm{c}}$ & 106 \\
\hline \multicolumn{3}{|l|}{ Non-infection } \\
\hline $\begin{array}{l}\text { Interstitial pneumonitis (non-infec- } \\
\text { tious) }\end{array}$ & $9(5 \%)$ & 178 \\
\hline ARDS & $8(4 \%)$ & 178 \\
\hline Thrombotic microangiopathy & $4(2 \%)$ & 178 \\
\hline Veno-occlusive disease & $8(4 \%)$ & 178 \\
\hline Central nervous system disorder & $3(2 \%)$ & 178 \\
\hline Hemorrhage & $7(4 \%)$ & 178 \\
\hline Bronchiolitis obliterans & $1(0.6 \%)$ & 178 \\
\hline Malignancy (NHL) & $2(1 \%)$ & 178 \\
\hline Short stature $(\leq-2 \mathrm{SD})$ & $24(22 \%)$ & 110 \\
\hline Gonadal dysfunction & $3(9 \%)$ & 33 \\
\hline Hypothyroidism & $4(7 \%)$ & 56 \\
\hline
\end{tabular}

${ }^{a}$ Including those who were diagnosed to have an infection before $\mathrm{HCT}$, after HCT, and the date of diagnosis is not available

${ }^{\mathrm{b}}$ The number (and percentage) of each item on the left is shown

${ }^{\mathrm{c}}$ Including only those who were diagnosed to have an infection after HCT

$C M V$, cytomegalovirus; $R S V$, respiratory syncytial virus; $E B V$, Epstein-Barr virus; $H H V 6$, human herpesvirus 6; VZV, varicella zoster virus; $A R D S$, acute respiratory distress syndrome; $N H L$, nonHodgkin lymphoma; $S D$, standard deviation

similar to our study. However, another study analyzing SCID patients from Eurocord [8] demonstrated cumulative incidences of $34 \% \pm 6 \%$ for grades II-IV aGVHD and $22 \% \pm 5 \%$ for cGVHD in UCBT recipients, which are higher than our cohort. These findings, which showed a similar HLA matching but less frequent GVHD in Japanese than in Western countries, imply that DPB1, DQB1, other minor histocompatibility antigens, or non-HLA gene polymorphisms, including drug metabolism, are probably more homogenous in the Japanese population, as suggested previously [17, 19]. Unsurprisingly, greater mismatch in UCB was associated with worse OS and a tendency for unstable engraftment. While HCT from MCB produced good results, caution must be exercised when HCT from $\mathrm{mMCB}$ is performed.

Regarding the conditioning regimen, no conditioning/IS had a negative impact on survival in this study. Considering that SCID-X1 and ADA-SCID are known to achieve great outcomes after HCT from MSDs without conditioning [20, 21], conditioning is often avoided for these patients with active infections at HCT. In this study, patients with such infections tended to receive unconditioned HCT, and death from infection was associated with not receiving conditioning. Therefore, the risk for no conditioning/IS may have been caused by concurrent active infections, which also worsened prognosis. Moreover, from 2006-2016, nine patients without active infection at HCT received no conditioning/IS, but four eventually died. We could not find a consistent trend for this mortality. Therefore, we cannot exclude the possibility that this small number of deaths influenced the multivariate analysis, showing that no conditioning/IS had an "independent" impact on poor OS.

In this study, we showed that post-transplant VOD and short stature were more common in the $\mathrm{Bu} / \mathrm{CY}$ regimen, implying toxicity of myeloablative busulfan doses, particularly in combination with a second alkylating agent. Short stature was relatively more common in our cohort than in another cohort (14\%) [22]. However, we speculate that conditioning was not a major cause of short stature for some patients because of the association between extensive cGVHD and short stature and four patients with documented short stature received no conditioning. We did not find any differences in outcomes between the Flu/Bu and $\mathrm{Flu} / \mathrm{Mel}$ regimens. Busulfan-containing regimens have been reported to be significantly associated with normal B cell function as compared with other regimens [23]. However, those who received busulfan-containing regimens exhibited an increased incidence of transplant-related late effects, such as gonadal dysfunction and infertility, which occurred more often than in the Flu/Mel regimens [24, 25]. Since the optimal conditioning regimen for SCID patients without MSD remains controversial, studies on the pharmacokinetics and pharmacodynamics of conditioning agents, studies on limiting doses of alkylating agents, and development of non-chemotherapeutic agents are being conducted with high priority [26]. The prognosis of $\mathrm{T}-\mathrm{B}+\mathrm{SCID}$ patients after HCT was good with low-dose busulfan $(30 \mathrm{mg} \cdot \mathrm{h} / \mathrm{L})$ combined with fludarabine [27]. Ongoing clinical trials determining the optimal doses of busulfan (NCT03619551) or anti-CD117 (NCT02963064) will also help to refine the 
guidelines on the optimal conditioning regimens for SCID. Furthermore, haploidentical transplantation with ex vivo T cell receptor $\alpha \beta$ and CD19 depletion [28-30] or lentiviral gene therapy [31,32] is reportedly feasible and efficacious for SCID patients. These novel methods will undoubtedly be important for developing future treatment strategies.

As in other large studies [11,13], active infection at HCT and later age of HCT were associated with poor survival in this study. Active bacterial or fungal infection at HCT correlated with a shorter interval between diagnosis and HCT. However, we do not have any data in the registry to clearly explain this result. In our cohort, although the median time between diagnosis and HCT was almost equal to that in North America, the median age at diagnosis was 5 months, which is later than that in North America, where NBS for SCID is available [33]. Therefore, once nationwide NBS is implemented, we anticipate that infections will be managed more appropriately and HCT will be performed at an earlier age, which should improve survival.

There are several limitations to this study. First, because our sample size is relatively small, we could not exclude bias. Second, the TRUMP did not contain busulfan pharmacokinetics, which reduced regimen-related toxicity analyses. Third, we did not evaluate immunological reconstitution and lineage-specific chimerism due to the lack of available information, which weakened our results and discussion. Fourth, we did not have NK cell status at the time of diagnosis. Future studies in Japan are expected to collect more specific PID information by interactively using the TRUMP and the Primary Immunodeficiency Database in Japan [34], which is currently in preparation.

In conclusion, we demonstrated that OS of HCT from MCB and MSD was similar, suggesting that a MCB donor can be an alternative for SCID patients without MSD. Better diagnostic methods for SCID, namely NBS, are also indispensable for better outcomes, as infection and later age at HCT were independent survival risks. In most areas in Japan, NBS is limited and availability should be expanded to improve prognosis after HCT.

Supplementary Information The online version contains supplementary material available at https://doi.org/10.1007/s10875-021-01112-5.

\footnotetext{
Acknowledgements We thank the Japan Marrow Donor Program, the cord blood banks in Japan, and the staff at the participating hospitals who attended to the patients and provided information for the TRUMP registry. We also thank Soichi Adachi, Shunichi Kato, Yasuo Horikoshi, Miharu Yabe, Nao Yoshida, Hiromitsu Takakura, Sae Ishimaru, Shinya Osone, Hidetoshi Takada, Nozomu Kawashima, Shinobu Tamura, Ayako Yamamori, Koji Kawaguchi, Risa Matsumura, and Takako Miyamura, who supported this study as members of the Hereditary Disorder Working Group of the Japanese Society for Transplantation and Cellular Therapy.
}

Authorship Contributions S.M. designed the research, analyzed the data, and wrote the manuscript. K.U., A.N., M.Y., H.Y., and K.K. revised the manuscript. M.Ku. and Y.A. verified the analytical method and analyzed the data. M.I., M.S., T.S., M.Kat., Y.S., A.I., T.K., Y.T., and M.Kaj. recruited the patients and collected the data. M.I., Y.H., and K.K. contributed to transplantation data management as members of the Japanese Data Center for Hematopoietic Cell Transplantation. K.I. and T.M. designed the research and revised the manuscript. All authors contributed to the article and approved the submitted version.

Funding This work was supported by the Japanese Ministry of Health, Labor, and Welfare [grant number 20FC1053] and the Japan Agency for Medical Research and Development [grant numbers JP19lk0201100 and JP19gk0110041].

Availability of Data and Material The datasets presented in this article are not readily available because the dataset belongs to the JSTCT and the Japanese Data Center for Hematopoietic Cell Transplantation (JDCHCT). Requests to access the datasets should be directed to http:// www.jdchct.or.jp/.

Code Availability All statistical analyses were performed using Stata software v16.1 and EZR 1.42.

\section{Declarations}

Ethics Approval The studies involving human participants were reviewed and approved by the Institutional Review Boards at the Japanese Society for Transplantation and Cellular Therapy (JSTCT) and Tokyo Medical and Dental University.

Consent to Participate/Publication All participants (and/or their guardians) provided written informed consent for research use of their data and publication.

Competing Interests The authors declare no competing interest.

\section{References}

1. Gaspar HB, Qasim W, Davies EG, Rao K, Amrolia PJ, Veys P. How I treat severe combined immunodeficiency. Blood. 2013;122:3749-58. https://doi.org/10.1182/ blood-2013-02-380105.

2. Tangye SG, Al-Herz W, Bousfiha A, Chatila T, CunninghamRundles C, Etzioni A, et al. Human inborn errors of immunity: 2019 update on the classification from the International Union of Immunological Societies Expert Committee. J Clin Immunol. 2020;40:24-64. https://doi.org/10.1007/s10875-020-00758-x.

3. Gatti RA, Meuwissen HJ, Allen HD, Hong R, Good RA. Immunological reconstitution of sex-linked lymphopenic immunological deficiency. Lancet. 1968;2:1366-9. https://doi.org/10.1016/ s0140-6736(68)92673-1.

4. Morio T, Atsuta Y, Tomizawa D, Nagamura-Inoue T, Kato K, Ariga T, et al. Outcome of unrelated umbilical cord blood transplantation in 88 patients with primary immunodeficiency in Japan. Br J Haematol. 2011;154:363-72. https://doi.org/10.1111/j.13652141.2011.08735.x.

5. Tsuji Y, Imai K, Kajiwara M, Aoki Y, Isoda T, Tomizawa D, et al. Hematopoietic stem cell transplantation for 30 patients with primary immunodeficiency diseases: 20 years experience of a single 
team. Bone Marrow Transplant. 2006;37:469-77. https://doi.org/ 10.1038/sj.bmt.1705273.

6. Iguchi A, Kawamura N, Kobayashi R, Takezaki S-I, Ohkura Y, Inamoto J, et al. Successful reduced-intensity SCT from unrelated cord blood in three patients with X-linked SCID. Bone Marrow Transplant. 2011;46:1526-31. https://doi.org/10.1038/bmt.2010. 338.

7. Kumaki S, Sasahara Y, Kamachi Y, Muramatsu H, Morio T, Goi $\mathrm{K}$, et al. B-cell function after unrelated umbilical cord blood transplantation using a minimal-intensity conditioning regimen in patients with X-SCID. Int J Hematol. 2013;98:355-60. https:// doi.org/10.1007/s12185-013-1408-7.

8. Fernandes JF, Rocha V, Labopin M, Neven B, Moshous $\mathrm{D}$, Gennery AR, et al. Transplantation in patients with SCID: mismatched related stem cells or unrelated cord blood? Blood. 2012;119:2949-55. https://doi.org/10.1182/ blood-2011-06-363572.

9. Atsuta Y, Suzuki R, Yoshimi A, Gondo H, Tanaka J, Hiraoka A, et al. Unification of hematopoietic stem cell transplantation registries in Japan and establishment of the TRUMP System. Int J Hematol. 2007;86:269-74. https://doi.org/10.1532/IJH97. 06239 .

10. Bousfiha A, Jeddane L, Picard C, Ailal F, Gaspar HB, Al-Herz W, et al. The 2017 IUIS Phenotypic Classification for Primary Immunodeficiencies. J Clin Immunol. 2017;38:129-43. https:// doi.org/10.1007/s10875-017-0465-8.

11. Pai S-Y, Logan BR, Griffith LM, Buckley RH, Parrott RE, Dvorak CC, et al. Transplantation outcomes for severe combined immunodeficiency, 2000-2009. N Engl J Med. 2014;371:43446. https://doi.org/10.1056/NEJMoa1401177.

12. Haddad E, Logan BR, Griffith LM, Buckley RH, Parrott RE, Prockop SE, et al. SCID genotype and 6-month posttransplant CD4 count predict survival and immune recovery. Blood. 2018;132:1737-49. https://doi.org/10.1182/ blood-2018-03-840702.

13. Gennery AR, Slatter MA, Grandin L, Taupin P, Cant AJ, Veys $\mathrm{P}$, et al. Transplantation of hematopoietic stem cells and longterm survival for primary immunodeficiencies in Europe: entering a new century, do we do better? J Allergy Clin Immunol. 2010;126:602-10. https://doi.org/10.1016/j.jaci.2010.06.015.

14. Kanda Y. Investigation of the freely available easy-to-use software "EZR" for medical statistics. Bone Marrow Transplant. 2013;48:452-8. https://doi.org/10.1038/bmt.2012.244.

15. Ikinciogullari A, Cagdas D, Dogu F, Tugrul T, Karasu G, Haskologlu S, et al. Clinical features and HSCT outcome for SCID in Turkey. J Clin Immunol. 2019;39:316-23. https://doi. org/10.1007/s10875-019-00610-x.

16. Mitchell R, Nivison-Smith I, Anazodo A, Tiedemann K, Shaw $\mathrm{P}$, Teague L, et al. Outcomes of hematopoietic stem cell transplantation in primary immunodeficiency: a report from the Australian and New Zealand Children's Haematology Oncology Group and the Australasian Bone Marrow Transplant Recipient Registry. Biol Blood Marrow Transplant. 2013;19:338-43. https://doi.org/10.1016/j.bbmt.2012.11.619.

17. Kanda J, Brazauskas R, Hu Z-H, Kuwatsuka Y, Nagafuji K, Kanamori H, et al. Graft-versus-host disease after HLA-matched sibling bone marrow or peripheral blood stem cell transplantation: comparison of North American Caucasian and Japanese populations. Biol Blood Marrow Transplant. 2016;22:744-51. https://doi.org/10.1016/j.bbmt.2015.12.027.

18. Eapen M, Wang T, Veys P, Boelens J, St Martin A, Spellman $S$, et al. Allele-level HLA matching for umbilical cord blood transplantation for non-malignant diseases in children: a retrospective analysis. Lancet Haematol. 2017;4:e325-33. https:// doi.org/10.1016/S2352-3026(17)30104-7.

19. Morishima S, Ogawa S, Matsubara A, Kawase T, NannyaYmKashiwase K, et al. Impact of highly conserved HLA haplotype on acute graft-versus-host disease. Blood. 2010;115:4664-740. https://doi.org/10.1182/blood-2009-10-251157.

20. Antoine C, Müller S, Cant A, Cavazzana-Calvo M, Veys P, Vossen $\mathrm{J}$, et al. Long-term survival and transplantation of haemopoietic stem cells for immunodeficiencies: report of the European experience 1968-99. Lancet. 2003;361:553-60. https://doi.org/10.1016/s0140-6736(03)12513-5.

21. Hassan A, Booth C, Brightwell A, Allwood Z, Veys P, Rao R, et al. Outcome of hematopoietic stem cell transplantation for adenosine deaminase-deficient severe combined immunodeficiency. Blood. 2012;120:3615-24-quiz3626. https://doi.org/10. 1182/blood-2011-12-396879.

22. Abd Hamid IJ, Slatter MA, McKendrick F, Pearce MS, Gennery AR. Long-term outcome of hematopoietic stem cell transplantation for IL2RG/JAK3 SCID: a cohort report. Blood. 2017;129:2198-201. https://doi.org/10.1182/ blood-2016-11-748616.

23. Haddad E, Leroy S, Buckley RH. B-cell reconstitution for SCID: should a conditioning regimen be used in SCID treatment? J Allergy Clin Immunol. 2013;131:994-1000. https:// doi.org/10.1016/j.jaci.2013.01.047.

24. Panasiuk A, Nussey S, Veys P, Amrolia P, Rao K, KrawczukRybak M, et al. Gonadal function and fertility after stem cell transplantation in childhood: comparison of a reduced intensity conditioning regimen containing melphalan with a myeloablative regimen containing busulfan. $\mathrm{Br} \mathrm{J}$ Haematol. 2015;170:719-26. https://doi.org/10.1111/bjh.13497.

25. Allewelt H, El-Khorazaty J, Mendizabal A, Taskindoust M, Martin PL, Prasad V, et al. Late effects after umbilical cord blood transplantation in very young children after busulfanbased, myeloablative conditioning. Biol Blood Marrow Transplant. 2016;22:1627-35. https://doi.org/10.1016/j.bbmt.2016. 05.024 .

26. Heimall J, Puck J, Buckley R, Fleisher TA, Gennery AR, Neven $\mathrm{B}$, et al. Current knowledge and priorities for future research in late effects after hematopoietic stem cell transplantation (HCT) for severe combined immunodeficiency patients: a consensus statement from the Second Pediatric Blood and Marrow Transplant Consortium International Conference on Late Effects after Pediatric HCT. Biol Blood Marrow Transplant. 2017;23:37987. https://doi.org/10.1016/j.bbmt.2016.12.619.

27. Nishimura A, Aoki Y, Ishiwata Y, Ichimura T, Ueyama J, Kawahara Y, et al. Hematopoietic cell transplantation with reduced intensity conditioning using fludarabine/busulfan or fludarabine/melphalan for primary immunodeficiency diseases. J Clin Immunol. 2021;41:944-57. https://doi.org/10.1007/ s10875-021-00966-z.

28. Balashov D, Shcherbina A, Maschan M, Trakhtman P, Skvortsova Y, Shelikhova L, et al. Single-center experience of unrelated and haploidentical stem cell transplantation with TCR $\alpha \beta$ and CD19 depletion in children with primary immunodeficiency syndromes. Biol Blood Marrow Transplant. 2015;21:1955-62. https://doi.org/10.1016/j.bbmt.2015.07.008.

29. Shah RM, Elfeky R, Nademi Z, Qasim W, Amrolia P, Chiesa $\mathrm{R}$, et al. T-cell receptor $\alpha \beta+$ and CD19+ cell-depleted haploidentical and mismatched hematopoietic stem cell transplantation in primary immune deficiency. J Allergy Clin Immunol. 
2018;141:1417-1426.e1. https://doi.org/10.1016/j.jaci.2017.07. 008.

30. Elfeky R, Shah RM, Unni MNM, Ottaviano G, Rao K, Chiesa R, et al. New graft manipulation strategies improve the outcome of mismatched stem cell transplantation in children with primary immunodeficiencies. J Allergy Clin Immunol. 2019;144:28093. https://doi.org/10.1016/j.jaci.2019.01.030.

31. De Ravin SS, Wu X, Moir S, Anaya-O'Brien S, Kwatemaa N, Littel P, et al. Lentiviral hematopoietic stem cell gene therapy for X-linked severe combined immunodeficiency. Sci Transl Med. 2016;8:335ra57. https://doi.org/10.1126/scitranslmed. aad8856.

32. Mamcarz E, Zhou S, Lockey T, Abdelsamed H, Cross SJ, Kang $\mathrm{G}$, et al. Lentiviral gene therapy combined with low-dose busulfan in infants with SCID-X1. N Engl J Med. 2019;380:1525-34. https://doi.org/10.1056/NEJMoa1815408.
33. Heimall J, Logan BR, Cowan MJ, Notarangelo LD, Griffith LM, Puck JM, et al. Immune reconstitution and survival of 100 SCID patients post-hematopoietic cell transplant: a PIDTC natural history study. Blood. 2017;130:2718-27. https://doi.org/10.1182/ blood-2017-05-781849.

34. Burrows PD, Fischer A. Building networks for immunodeficiency diseases and immunology training. Nat Immunol. 2008;9:1005-7. https://doi.org/10.1038/ni0908-1005.

Publisher's Note Springer Nature remains neutral with regard to jurisdictional claims in published maps and institutional affiliations.

\section{Authors and Affiliations}

\section{Satoshi Miyamoto ${ }^{1,2} \cdot$ Katsutsugu Umeda $^{2,3} \cdot$ Mio Kurata $^{4} \cdot$ Akira Nishimura $^{1,2} \cdot$ Masakatsu Yanagimachi $^{2,5}$.

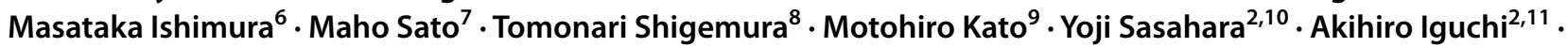 Takashi Koike $^{12} \cdot$ Yoshiyuki Takahashi $^{13} \cdot$ Michiko Kajiwara $^{14} \cdot$ Masami Inoue $^{7} \cdot$ Yoshiko Hashii $^{15} \cdot$ Hiromasa Yabe $^{2,16}$. Koji Kato $^{2,17} \cdot$ Yoshiko Atsuta ${ }^{4,18} \cdot$ Kohsuke Imai $^{2,19}$ (Domohiro Morio ${ }^{1,2}$}

1 Department of Pediatrics and Developmental Biology, Tokyo Medical and Dental University, 1-5-45 Yushima, Bunkyo-ku, Tokyo, Japan

2 Hereditary Disorder Working Group of the Japanese Society for Transplantation and Cellular Therapy, 1-1-20 Daiko-Minami, Higashi-ku, Nagoya, Aichi, Japan

3 Department of Pediatrics, Graduate School of Medicine, Kyoto University, 54 Kawaharacho, Shogoin, Sakyo-ku, Kyoto, Japan

4 Japanese Data Center for Hematopoietic Cell Transplantation, 1-1-20 Daiko-Minami, Higashi-ku, Nagoya, Aichi, Japan

5 Division of Hematology/Oncology, Kanagawa Children's Medical Center, 2-138-4 Mutsukawa, Minami-ku, Yokohama, Kanagawa, Japan

6 Department of Pediatrics, Graduate School of Medical Sciences, Kyushu University, 3-1-1 Maidashi, Higashi-ku, Fukuoka, Japan

7 Department of Hematology/Oncology, Osaka Women's and Children's Hospital, 840 Murodocho, Izumi, Osaka, Japan

8 Department of Pediatrics, Shinshu University School of Medicine, 3-1-1 Asahi, Matsumoto, Nagano, Japan

9 Children's Cancer Center, National Center for Child Health and Development, 2-10-1 Okura, Setagaya-ku, Tokyo, Japan

10 Department of Pediatrics, Tohoku University Graduate School of Medicine, 1-1 Seiryo-machi, Aoba-ku, Sendai, Miyagi, Japan
11 Department of Pediatrics, Hokkaido University Hospital, Kita14, Nishi5, Kita-Ku, Sapporo, Hokkaido, Japan

12 Department of Pediatrics, Tokai University School of Medicine, 143 Shimokasuya, Isehara, Kanagawa, Japan

13 Department of Pediatrics, Nagoya University Graduate School of Medicine, 65 Tsurumaicho, Showa-ku, Nagoya, Aichi, Japan

14 Center for Transfusion Medicine and Cell Therapy, Tokyo Medical and Dental University, Medical Hospital, 1-5-45 Yushima, Bunkyo-ku, Tokyo, Japan

15 Department of Cancer Immunotherapy, Osaka University Graduate School of Medicine, Yamadaoka, Suita, Osaka 2-15, Japan

16 Department of Innovative Medical Science, Tokai University School of Medicine, 143 Shimokasuya, Isehara, Kanagawa, Japan

17 Central Japan Cord Blood Bank, 539-3 Minami-Yamaguchi-cho, Aichi Red Cross Blood Center 4F, Seto, Aichi, Japan

18 Department of Registry Science for Transplant and Cellular Therapy, Aichi Medical University School of Medicine, 1-1 Yazakokarimata, Nagakute, Aichi, Japan

19 Department of Community Pediatrics, Perinatal, and Maternal Medicine, Tokyo Medical and Dental University, 1-5-45 Yushima, Bunkyo-ku, Tokyo 113-8519, Japan 